\title{
Title: Effects of Small-Angle Mistilts on Dopant Visibility in
}

\section{ADF-STEM Imaging of Nanocrystals}

Authors: Jacob T. Held, Samuel Duncan, K. Andre Mkhoyan*

Affiliation: Department of Chemical Engineering and Materials Science, University of Minnesota, Minneapolis, Minnesota 55455, USA

*To whom correspondence may be addressed:

K. Andre Mkhoyan: Email. mkhoyan@umn.edu; Tel.612-625-2059

\begin{abstract}
:
Quantitative ADF-STEM imaging paired with image simulations has proven to be a powerful technique for determining the three dimensional location of substitutionally doped atoms in thin films. Expansion of this technique to lightly-doped nanocrystals requires an understanding of the influence of specimen mistilt on dopant visibility due to the difficulty of accurate orientation determination in such systems as well as crystal movement under the beam. In this study, the effects of specimen mistilt on ADF-STEM imaging are evaluated using germanium-doped silicon nanocrystals as model systems. It is shown that dopant visibility is a strong function of specimen mistilt, and the accuracy of specimen orientation is an important factor in the analysis of three-dimensional dopant location, but the sensitivity to mistilt can be
\end{abstract}


weakened by increasing the STEM probe convergence angle and optimizing ADF detector inner angle.

\section{Introduction}

Many electronic devices rely on the incorporation of dopants in semiconductors to tune semiconductor properties. In nanocrystals and nanoscale devices, these properties can become highly dependent on the location and coordination of discrete dopant atoms, necessitating characterization with single atom sensitivity [1,2]. Annular dark-field scanning transmission electron microscopy (ADF-STEM) has been demonstrated to be a powerful technique for visualizing of individual substitutionally doped atoms in both nanoparticles and films [3]. Provided the dopant has a considerably higher atomic number than its host, dopant atoms can be clearly visible in ADF-STEM images and readily located to an atomic column, especially in thin, lightly doped specimens [4-8]. However, localization of individual dopant atoms in the third dimension, or depth, is much more challenging.

Quantitative ADF-STEM imaging of substitutionally doped specimens has drawn considerable attention recently for 3D dopant location, especially in beam-sensitive materials, because it requires only a single image rather than the large series of images necessary for focal series [9] and tomography [10,11]. This method utilizes the effects of electron beam channeling down the atomic columns, which produces a measurable and predictable relationship between dopant depth and the intensity of the dopant-containing column with respect to an equivalent non-doped column. This relationship has been used to infer the depth of a dopant atom along an atomic column [12,13]. A critical step of this analysis is the precise determination of specimen thickness and crystallographic orientation. These parameters have been primarily determined by 
comparing quantitative measurements of ADF-STEM signals and position averaged convergent beam electron diffraction (PACBED) patterns with their corresponding simulations. This approach for thickness and orientation determination works well for thin, single-crystal films, where experimental PACBED has been shown to determine specimen tilt to within $\pm 1 \mathrm{mrad}$ [14].

However, because of specimen damage and the inherent thickness variation due to the geometry of a nanocrystal specimen, it is often difficult to obtain quantifiable PACBED patterns from small nanocrystals. This leaves only high-resolution ADF-STEM imaging of the crystal lattice available for orientation determination [15]. In the case of small $(2-5 \mathrm{~nm})$ nanocrystals oriented near a low-order zone axis, specimen orientation may only be approximated through HAADF imaging alone to within about $+/$ - 30 mrad. As we observed from a set of simulations conducted for different beam voltages and convergence angles, a sample of which are shown in Figure 1, mistilts away from zone axis less than about $+/-30 \mathrm{mrad}$ become qualitatively difficult to distinguish from residual beam aberrations. It is, therefore, important to understand and evaluate the effects of specimen mistilt from zone axis on dopant visibility in order to determine dopant locations in the third dimension, or depth, through quantitative ADF-STEM.

In this work, built on literature discussing the effects of specimen tilt on atomicresolution ADF-STEM images of undoped crystals [15-20], the effect of inaccuracies in alignment near low-order zone axes on the visibility of dopant atoms in ADF-STEM images has been computationally investigated for nanocrystals. Here, simple $2.5 \mathrm{~nm}$ Ge-doped $\mathrm{Si}$ nanocrystals were used as a study system. Si is a common and well-characterized material and Ge dopants are preferentially located into substitutional sites. In order to isolate the effects of mistilt from other sources of experimental error, surface reconstruction and non-uniformity and residual aberrations of the lenses are ignored in these simulations. While this study examines an 
ideal, singly-doped spherical nanocrystal, the effects that are demonstrated can be extended into thin films and other specimen geometries and experimental setups.

\section{Methods}

ADF-STEM images of Ge-doped Si nanocrystals were simulated through the TEMSIM multislice package [21]. This method has been previously used to understand beam channeling, quantitative STEM, and dopant visibility [15,22-25]. Spherical models of $2.5 \mathrm{~nm}$ in diameter $\mathrm{Si}$ nanocrystals were generated by replicating the Si lattice out to a maximum radius of $1.25 \mathrm{~nm}$. The structures were then rotated to the [001], [110], and [111] zone axes, and Ge dopants were iteratively substituted into lattice sites of the central column to form singly-doped structures. For the case studied here, the difference between atomic sizes of the dopant $\mathrm{Ge}$ and host $\mathrm{Si}\left(\mathrm{R}_{\mathrm{Ge}}=\right.$ $1.22 \AA$ and $\mathrm{R}_{\mathrm{Si}}=1.18 \AA$ ) is very small relative the Si-Si bond length and, therefore, the effects of strain introduced by the dopant can be ignored $[22,26]$. However, it is not always the case $[8,27,28]$ and structural relaxation might be needed in other systems before applying multislice code.

For simulated ADF-STEM images of entire particles, the STEM converged probe was scanned over a $30 \times 30 \AA^{2}$ area containing the nanocrystal with 256 x 256 pixels. For detailed analysis of all cases, the converged probe was scanned over an $8.33 \times 8.33 \AA^{2}$ area in the center of the nanocrystal with $85 \times 85$ pixels. In each case, a transmission function was calculated for a 30 x $30 \AA^{2}$ supercell with 1024 x 1024 pixels. ADF-STEM images were calculated for 54/200 mrad inner/outer collection angles. The following slice thicknesses were used in all simulations: $1.357 \AA, 1.920 \AA$, and $0.783 \AA$ for beams propagating along the [001], [110], and [111] zone axes, respectively. The nanocrystals were tilted within the TEMSIM code from 0 to $30 \mathrm{mrad}$ off 
zone axis in 2 mrad steps using the slice shifting method which is detailed in Ref [21]. Due to the small size of the nanocrystals in this study, and the even smaller regions of interest, no statistically significant difference was observed between this approximation of specimen tilt and a rigorous manual tilting approach when studying specimens tilted up to $30 \mathrm{mrad}$ off zone axis.

To show that the observations made here are not unique to a single experimental setup, two common operating conditions for aberration-corrected STEM were considered in this study. First, an aberration corrected $100 \mathrm{kV}$ probe with a convergence angle of $\alpha_{\mathrm{obj}}=25 \mathrm{mrad}$, aberration coefficients of $\mathrm{C}_{\mathrm{s}(3)}=-0.015 \mathrm{~mm}, \mathrm{C}_{\mathrm{s}(5)}=10 \mathrm{~mm}$, and defocus of $\Delta \mathrm{f}=-30 \AA$ was used. For this probe, a $1 \AA$ FWHM Gaussian source distribution, typical for $100 \mathrm{kV}$ beam, was applied by convoluting the source distribution function into images [22]. Second, an aberration corrected $200 \mathrm{kV}$ probe with a convergence angle of $\alpha_{\mathrm{obj}}=25 \mathrm{mrad}$, zero aberrations were considered. For this probe a $0.5 \AA$ FWHM Gaussian source distribution, typical for $200 \mathrm{kV}$ beam, was applied [29]. Variations of this probe were also studied with $\alpha_{o b j}=35 \mathrm{mrad}$ and $45 \mathrm{mrad}$ and ADF inner/outer angles of 65/240 and 75/278 mrad, respectively. Thermal vibrations of atoms in the nanocrystals were included by averaging 100 frozen phonon configurations using the room temperature RMS displacements (vibration amplitudes) of $0.076 \AA$ for all Si atoms and $0.080 \AA$ for Ge dopants [22,30,31]. All ADF-STEM images were normalized to the intensity of the incident beam.

\section{Results and Discussion}

ADF-STEM images were generated for intrinsic as well as singly-doped nanocrystals with Ge atoms iteratively positioned at each available dopant site in the central column along each zone axis. Figure 1 shows example images of intrinsic, doped, and tilted doped samples that 
represent the most visible effects of tilting. As shown in these images, there is relatively little overall qualitative difference between the tilted and non-tilted samples. Additional effects that are not included in these simulations from statistical and instrumentation noise, probe aberrations, nanocrystal movement, and background from the support would make these differences virtually undetectable in experimentally recorded images. It should be noted that the range of the specimen mistilt that still produces practically indistinguishable high-resolution ADF-STEM images is function of specimen defined by the types of atoms it contains and spacing between them.

The dopant-containing columns appear brighter than the Si host due to the higher atomic number of Ge. This can be quantified by the dopant visibility, defined as:

$$
V=\frac{I_{D}-I_{I}}{I_{I}} * 100 \%
$$

where $I_{D}$ and $I_{I}$ are the $\mathrm{ADF}$ intensities of the dopant containing column and the equivalent intrinsic column, respectively [22]. Values of $I_{I}$ and $I_{D}$ were recorded from ADF-STEM images of dopant-containing columns and analogous intrinsic nanocrystals under the same simulation conditions. To obtain these intensities, each image was spline-interpolated to a pixel size of $0.01 \AA$ and the average intensity within a $0.25 \AA$ radius circle centered on the maximum intensity of the column was chosen, emulating the practical evaluation of the ADF intensity from experimental images [12]. While the evaluation radius defining $I_{D}$ and $I_{I}$ slightly effects the values of the intensities and visibilities, the overall relationships between dopant visibility and mistilt remain the same (tested for 0-0.75 $\AA$ radii; not shown here). 
(a)
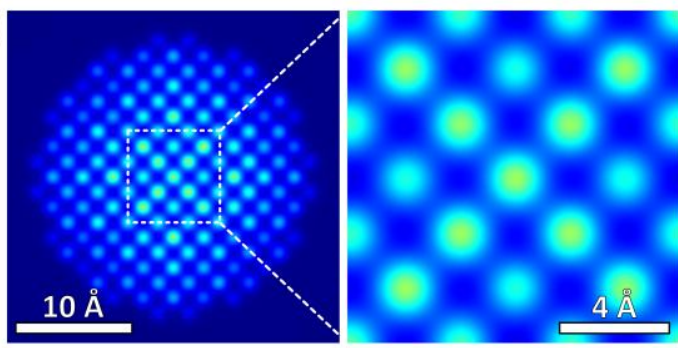

(b)
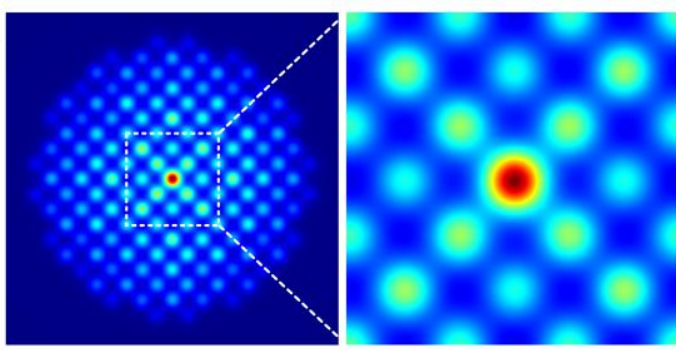

(c)
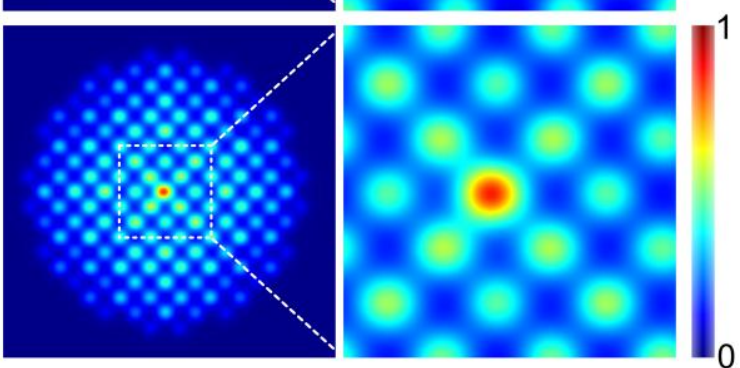

Figure 1. Example of a set of ADF-STEM images simulated for [001] oriented $2.5 \mathrm{~nm} \mathrm{Si}$ nanocrystals imaged at $100 \mathrm{kV}$ for three cases: (a) intrinsic nanocrystal, (b) nanocrystal with $\mathrm{Ge}$ dopant placed at the beam exit surface, and (c) the same doped nanocrystal as in (b) tilted 30 mrad off axis. All three images have been re-normalized here to the maximum intensity of the un-tilted doped particle in (b) for visual clarity. The enlarged subset regions of interest for each case are shown on the right. The apparent cross pattern of intensity arises from a variation in the number of atoms in each column due to the particle geometry.

A [001] oriented Ge-doped Si nanocrystal imaged with an aberration-corrected $100 \mathrm{kV}$ beam was considered first. The visibilities of Ge dopant atoms positioned at each of the five possible dopant sites down the central column were calculated for mistilts of 0-30 mrad off zone axis (Figure 2). At zero mistilt, we see a unique relationship between dopant depth and visibility, which could be used to determine dopant location to within one unit cell, given experimental visibility error is less than 5\%. However, mistilt complicates this system, with visibilities of dopants at different sites overlapping and changing order with increasing mistilt. 

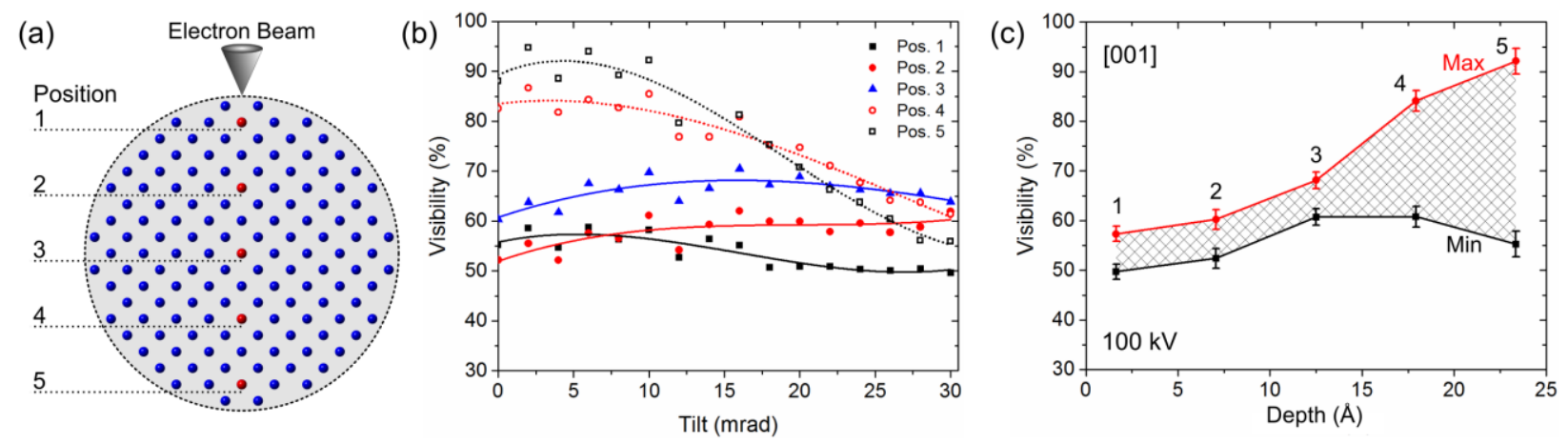

Figure 2. (a) A model of [001] oriented Si nanocrystal showing the positions of the five dopant sites in the central column. (b) Full set of simulated data showing the relationship between tilt and visibility for dopants in ADF-STEM images at each lattice sites of a dopant near the [001] zone axis. Cubic fits to the data are included to guide the eye. (c) Minimum and maximum visibilities at each dopant position in (b) showing the error in depth associated with a $30 \mathrm{mrad}$ tilt uncertainty. Error bars correspond to the standard deviation from the cubic fits shown in (b). Simulations were performed for aberration-corrected $100 \mathrm{kV}$ STEM.

When a crystal is oriented precisely to zone axis, electron channeling focuses the beam onto the column of atoms as it passes through the nanocrystal [32]. Due to this focusing effect, channeling causes dopants near the exit surface of the nanocrystal to exhibit much higher visibility $(\sim 90 \%)$ than dopants near the entrance surface $(\sim 55 \%)$. This channeling effect is reduced rapidly at mistilts over $\sim 15 \mathrm{mrad}$ because the beam is no longer propagating down a zone axis, causing the beam to broaden more as a function of depth and interact less with deeper atoms along that column $[9,15,22]$. This causes a significant reduction in dopant visibility near the exit surface and more complex behavior for dopants at intermediate sites, with some visibilities rising and some falling with increasing mistilt.

A cubic function was used to fit each tilt series to aid visualization of the relationship between dopant visibility and tilt and to provide an estimate of the computational error due to the finite number of phonons used in the simulations. The results of all simulations in this series are summarized in Figure 2c, where the minimum and maximum values of the fit functions for 
mistilts between 0 and 30 mrad are shown for each dopant position. The shaded region indicates the range of dopant visibility possible over mistilts of $0-30 \mathrm{mrad}$ off axis for each dopant position. The strong relationship between dopant visibility and mistilt results in considerable overlap between the potential visibilities of dopants in different positions over this range of mistilt. This, therefore, precludes the determination of 3D dopant location from a single image of such a nanocrystal with some mistilt. Additionally, under these conditions mistilt uncertainty of $<5 \mathrm{mrad}$ is enough to contribute non-negligibly to dopant visibility.

A similar sensitivity to mistilt was observed in ADF-STEM images of the same system imaged with the $200 \mathrm{kV}$ beam and convergence angle $\alpha_{\mathrm{obj}}=25 \mathrm{mrad}$ (Figure $3 \mathrm{a}$ ). In addition to [001] oriented samples, analogous simulations of [110] and [111] oriented nanocrystals were considered (Figure $3 \mathrm{~b}, \mathrm{c}$ ). The dopant visibility as a function of depth near these zone axes is a more complex function of mistilt than those near [001]. However, the overall trends remain same, with the visibility of dopants closest to the exit surface, which are most sensitive to channeling, exhibiting the greatest effect. In all considered cases, small mistilt near zone axis creates a strong enough effect on dopant visibility that no single visibility value can be unambiguously attributed to a single dopant position. 

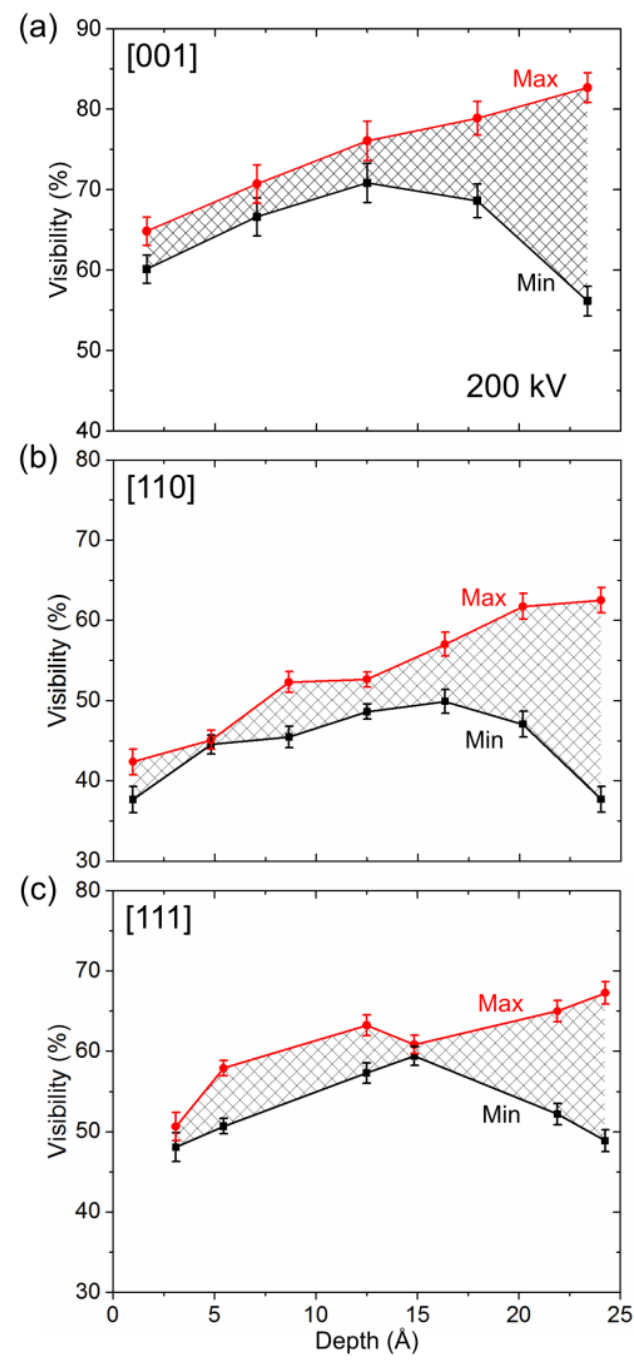

Figure 3. (a-c) Minimum and maximum visibilities of dopants in ADF-STEM images due to 0 to $30 \mathrm{mrad}$ mistilt for Ge dopants in $2.5 \mathrm{~nm}$ Si nanocrystal at each possible lattice site in the central column for [001], [110], and [111] zone axes oriented nanocrystals. Simulations were performed for aberration-corrected $200 \mathrm{kV}$ STEM with convergence angle $\alpha_{\mathrm{obj}}=25 \mathrm{mrad}$.

It is possible to influence the tilt sensitivity of dopant visibility by modifying the convergence angle and ADF detector inner angle. In this case, increasing the convergence angle of the STEM probe above 25 mrad allows a reduction of probe depth of field, resulting in a substantial reduction in mistilt sensitivity. The results for 35 and $45 \mathrm{mrad}$ probe convergence angles are presented in Figure 4. As can be seen from the figure, when probe convergence angle 
is increased to $45 \mathrm{mrad}$, the location of a single substitutional Ge dopant can be determined to within one unit cell, barring other sources of error. These results indicate that increasing STEM probe convergence angle can be used to mitigate the uncertainties in dopant location determination due to mistilt.
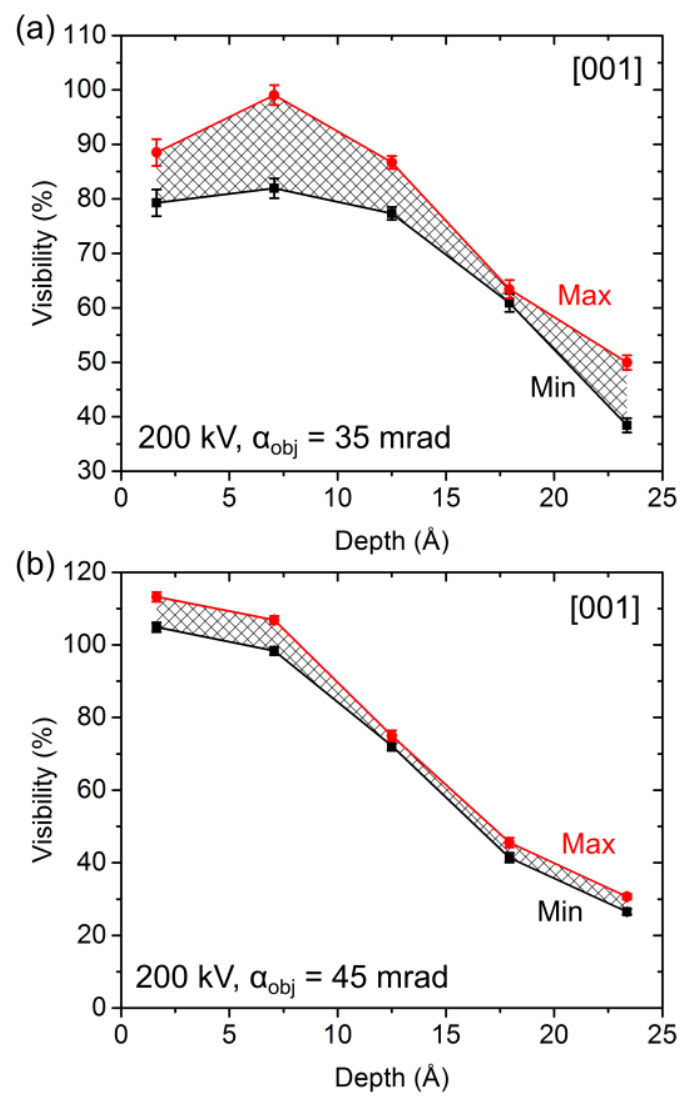

Figure 4. Minimum and maximum visibilities of dopants in ADF-STEM images due to 0 to 30 mrad mistilt for Ge dopants in $2.5 \mathrm{~nm}$ Si nanocrystal at each lattice site in the central column for [001] oriented nanocrystals. Simulations were performed for aberration-corrected $200 \mathrm{kV}$ STEM with convergence angles: (a) $\alpha_{\mathrm{obj}}=35 \mathrm{mrad}$, ADF inner/outer angles 65/240 mrad and (b) $\alpha_{\mathrm{obj}}=$ $45 \mathrm{mrad}$, ADF inner/outer angles 75/278 mrad. ADF detector outer angles were scaled proportionally with the inner angles to emulate an experimental detector.

To understand the possible effects of varying ADF detector inner angle, we evaluated the difference in visibility of the Ge dopant located at near the exit surface (most sensitive case) as a function of $\mathrm{ADF}$ detector inner angle for 0 and $30 \mathrm{mrad}$ mistilts. These simulations were 
performed for $\alpha_{\mathrm{obj}}=25,35$ and $45 \mathrm{mrad}$ probe convergence angles at $200 \mathrm{kV}$. As shown in Figure 5, increasing ADF inner angle can yield modest improvements in the mitigation of dopant visibility sensitivity to mistilt at convergence angles below $45 \mathrm{mrad}$. However, it should be noted that increasing $\mathrm{ADF}$ inner angle also reduces the ADF signal, which is not desirable.

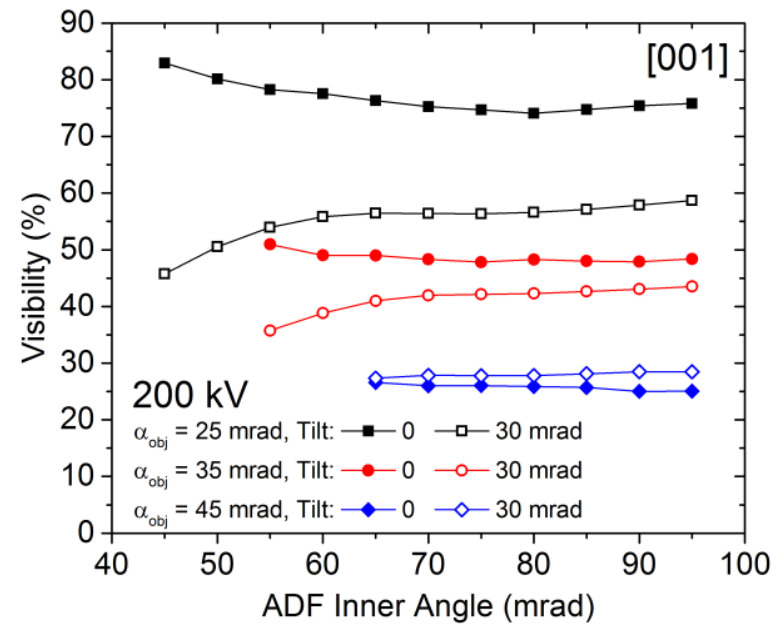

Figure 5. ADF inner angle dependence of dopant visibility at different STEM probe convergence angles for the [001] oriented nanocrystal doped near the exit surface (position $\# 5$ from Figure 1). In these simulations, the ADF detector outer angles were scaled proportionally with the inner angles to emulate an experimental detector.

Because of the small size of the nanocrystals investigated here and the positioning of dopants along the central column of the nanocrystals, sample geometry contributed negligibly to probe behavior in the sample. Additionally, the inclusion of a source distribution resulted in slight changes in dopant visibility compared with zero source size, but no notable differences in the visibility trends were observed. For these reasons, it is expected that other samples and experimental settings will also exhibit similar sensitivity of dopant visibility to specimen mistilt.

These results show that even small mistilt error can contribute non-negligibly to dopant visibility under normal imaging conditions. These effects may be mitigated through the use of a higher convergence angle probe and optimization of the ADF inner angle, but the effects of 
mistilt should still be carefully considered as a non-negligible source of visibility error. However, given adequate control of specimen tilt, it is possible that this tilt dependence may be utilized as an additional parameter in dopant location identification because over the range of structures analyzed, each dopant position exhibited a unique relationship between dopant visibility and small specimen mistilts, with the greatest effect occurring at low convergence angles and voltages.

\section{Conclusion}

The results of multislice simulation of ADF-STEM images of Ge-doped Si nanocrystals suggest that specimen mistilt near zone axis can significantly influence dopant visibility. In the system studied, visibility changes over mistilts between 0 - $30 \mathrm{mrad}$ are large enough to preclude accurate 3D dopant location with a $25 \mathrm{mrad}$ probe, and in some cases, even mistilt of less than 5 mrad can contribute non-negligibly to dopant visibility. Increasing the probe convergence angle, and ADF detector inner angle at some degree, can be used to mitigate mistilt sensitivity of dopant visibility. While this study explicitly considered nanocrystals, we expect that the sensitivity of dopant visibility to misorientation extends to more complex systems as well as thin films.

\section{Acknowledgements}

This project was supported by the MRSEC program of the National Science Foundation under Award Number DMR-1420013. This project was also partially supported by the University of Minnesota Undergraduate Research Opportunities Program (UROP). The authors acknowledge the Minnesota Supercomputing Institute (MSI) at the University of Minnesota for providing resources that contributed to the research results reported within this project. 


\section{References}

[1] P.M. Koenraad, M.E. Flatté, Single dopants in semiconductors., Nat. Mater. 10 (2011) 91100. doi:10.1038/nmat2940.

[2] D.J. Norris, A.L. Efros, S.C. Erwin, Doped nanocrystals., Science. 319 (2008) 1776-1779. doi:10.1126/science. 1143802 .

[3] V.C. Holmberg, J.R. Helps, K.A. Mkhoyan, D.J. Norris, Imaging impurities in semiconductor nanostructures, Chem. Mater. 25 (2013) 1332-1350.

[4] P.M. Voyles, D. a Muller, J.L. Grazul, P.H. Citrin, H.-J.L. Gossmann, Atomic-scale imaging of individual dopant atoms and clusters in highly n-type bulk Si., Nature. 416 (2002) 826-829.

[5] P.M. Voyles, J.L. Grazul, D. a. Muller, Imaging individual atoms inside crystals with ADF-STEM, Ultramicroscopy. 96 (2003) 251-273. doi:10.1016/S0304-3991(03)00092-5.

[6] H. Okuno, J.-L. Rouvière, P.-H. Jouneau, P. Bayle-Guillemaud, B. Daudin, Visualization of Tm dopant atoms diffused out of GaN quantum dots, Appl. Phys. Lett. 96 (2010) 251908. doi:10.1063/1.3455893.

[7] M.D. Rossell, Q.M. Ramasse, S.D. Findlay, F. Rechberger, R. Erni, M. Niederberger, Direct imaging of dopant clustering in metal-oxide nanoparticles, ACS Nano. 6 (2012) 7077-7083.

[8] N. Shibata, S.D. Findlay, S. Azuma, T. Mizoguchi, T. Yamamoto, Y. Ikuhara, Atomicscale imaging of individual dopant atoms in a buried interface., Nat. Mater. 8 (2009) 654658. doi:10.1038/nmat2486.

[9] H.L. Xin, V. Intaraprasonk, D. a. Muller, Depth sectioning of individual dopant atoms with aberration-corrected scanning transmission electron microscopy, Appl. Phys. Lett. 92 (2008) 13125. doi:10.1063/1.2828990.

[10] S. Bals, B. Goris, A. De Backer, S. Van Aert, G. Van Tendeloo, Atomic resolution electron tomography, MRS Bull. 41 (2016) 525-530. doi:10.1557/mrs.2016.138.

[11] S. Van Aert, K.J. Batenburg, M.D. Rossell, R. Erni, G. Van Tendeloo, Three-dimensional atomic imaging of crystalline nanoparticles., Nature. 470 (2011) 374-377.

doi:10.1038/nature09741.

[12] J. Hwang, J.Y. Zhang, A.J. D’Alfonso, L.J. Allen, S. Stemmer, Three-dimensional imaging of individual dopant atoms in SrTiO3, Phys. Rev. Lett. 111 (2013) 1-5. doi:10.1103/PhysRevLett.111.266101.

[13] R. Ishikawa, A.R. Lupini, S.D. Findlay, T. Taniguchi, S.J. Pennycook, Three-dimensional location of a single dopant with atomic precision by aberration-corrected scanning transmission electron microscopy, Nano Lett. 14 (2014) 1903-1908. doi:10.1021/nl500564b.

[14] J.M. LeBeau, S.D. Findlay, L.J. Allen, S. Stemmer, Position averaged convergent beam electron diffraction: Theory and applications, Ultramicroscopy. 110 (2010) 118-125. 
doi:10.1016/j.ultramic.2009.10.001.

[15] S.E. Maccagnano-Zacher, K.A. Mkhoyan, E.J. Kirkland, J. Silcox, Effects of tilt on highresolution ADF-STEM imaging, Ultramicroscopy. 108 (2008) 718-726. doi:10.1016/j.ultramic.2007.11.003.

[16] T. Yamazaki, M. Kawasaki, K. Watanabe, I. Hashimoto, M. Shiojiri, Effect of small crystal tilt on atomic-resolution high-angle annular dark field STEM imaging, Ultramicroscopy. 92 (2002) 181-189. doi:10.1016/S0304-3991(02)00131-6.

[17] P. Wang, A.L. Bleloch, U. Falke, P.J. Goodhew, Geometric aspects of lattice contrast visibility in nanocrystalline materials using HAADF STEM, Ultramicroscopy. 106 (2006) 277-283. doi:10.1016/j.ultramic.2005.09.005.

[18] X. Wu, M.D. Robertson, M. Kawasaki, J.M. Baribeau, Effects of small specimen tilt and probe convergence angle on ADF-STEM image contrast of Si $0.8 \mathrm{Ge} 0.2$ epitaxial strained layers on (100) Si, Ultramicroscopy. 114 (2012) 46-55. doi:10.1016/j.ultramic.2012.01.001.

[19] Y.G. So, K. Kimoto, Effect of specimen misalignment on local structure analysis using annular dark-field imaging, J. Electron Microsc. (Tokyo). 61 (2012) 207-215. doi:10.1093/jmicro/dfs045.

[20] K.E. MacArthur, A.J. D’Alfonso, D. Ozkaya, L.J. Allen, P.D. Nellist, Optimal ADF STEM imaging parameters for tilt-robust image quantification, Ultramicroscopy. 156 (2015) 1-8. doi:10.1016/j.ultramic.2015.04.010.

[21] E.J. Kirkland, Advanced computing in electron microscopy: Second edition, 2nd ed., Springer Science + Business Media, New York, 2010. doi:10.1007/978-1-4419-6533-2.

[22] A. Mittal, K.A. Mkhoyan, Limits in detecting an individual dopant atom embedded in a crystal, Ultramicroscopy. 111 (2011) 1101-1110. doi:10.1016/j.ultramic.2011.03.002.

[23] R.F. Loane, E.J. Kirkland, J. Silcox, Visibility of single heavy atoms on thin crystalline silicon in simulated annular dark-field STEM images, Acta Crystallogr. Sect. A Found. Crystallogr. 44 (1988) 912-927. doi:10.1107/S0108767388006403.

[24] E.C. Cosgriff, A. J. D’Alfonso, L.J. Allen, S.D. Findlay, A. I. Kirkland, P.D. Nellist, Three-dimensional imaging in double aberration-corrected scanning confocal electron microscopy, Part I.. Elastic scattering, Ultramicroscopy. 108 (2008) 1558-1566. doi:10.1016/j.ultramic.2008.05.009.

[25] J.M. LeBeau, S.D. Findlay, L.J. Allen, S. Stemmer, Standardless atom counting in scanning transmission electron microscopy, Nano Lett. 10 (2010) 4405-4408. doi: $10.1021 / \mathrm{nl} 102025 \mathrm{~s}$.

[26] R.R. Vanfleet, M. Robertson, M. McKay, J. Silcox, Prospects for single atom sensitivity measurements of dopant levels in silicon, in: Charact. Metrol. ULSI Technol. 1998 Int. Conf., Asce, 1998: pp. 901-905. doi:10.1063/1.56884.

[27] D.A. Muller, N. Nakagawa, A. Ohtomo, J.L. Grazul, H.Y. Hwang, Atomic-scale imaging of nanoengineered oxygen vacancy profiles in SrTiO3., Nature. 430 (2004) 657-661. 
doi:10.1038/nature02772.1.

[28] J.S. Jeong, P. Ambwani, B. Jalan, C. Leighton, K.A. Mkhoyan, Observation of electrically-inactive interstitials in Nb-doped SrTiO 3, ACS Nano. 7 (2013) 4487-4494. doi:10.1021/nn401101y.

[29] M.L. Odlyzko, J.T. Held, K.A. Mkhoyan, Atomic bonding effects in annular dark field scanning transmission electron microscopy. II. Experiments, J. Vac. Sci. Technol. A Vacuum, Surfaces, Film. 34 (2016) 41603. doi:10.1116/1.4954877.

[30] R.F. Loane, P. Xu, J. Silcox, Thermal vibrations in convergent-beam electron diffraction, Acta Crystallogr. Sect. A. 47 (1991) 267-278. doi:10.1107/S0108767391000375.

[31] J.S. Reid, J.D. Pirie, Dynamic deformation and the Debye-Waller factors for silicon-like crystals, Acta Crystallogr. Sect. A. 36 (1980) 957-965. doi:10.1107/S0567739480001982.

[32] E.J. Kirkland, Some effects of electron channeling on electron energy loss spectroscopy, Ultramicroscopy. 102 (2005) 199-207. doi:10.1016/j.ultramic.2004.09.010. 
